\title{
A interação universidades/institutos públicos de pesquisa e empresas no Brasil: resultados comparativos entre o relacionamento com empresas nacionais e multinacionais
}

\author{
Fábio Chaves do Couto e Silva Neto ${ }^{1}$ \\ Ulisses Pereira dos Santos ${ }^{2}$ \\ Vanessa Parreiras Oliveira ${ }^{3}$ \\ Priscila Gomes de Castro ${ }^{4}$ \\ Luiza Teixeira de Melo Franco ${ }^{5}$ \\ Fernanda De Negri ${ }^{6}$
}

\begin{abstract}
Resumo: O objetivo deste artigo é investigar os padrões de interação de empresas nacionais e multinacionais localizadas no Brasil com universidades/institutos públicos de pesquisa (IPPs) presentes no país. Para tanto, utilizou-se uma base de dados que envolve 319 questionários respondidos pelos responsáveis pelas atividades de pesquisa e desenvolvimento (P\&D) e/ou pelas interações com universidades e IPPs em empresas localizadas no Brasil, as quais apresentavam interação com grupos de pesquisa sediados em universidades/IPPs brasileiros no ano de 2004. Tais informações foram avaliadas por meio de uma análise descritiva dos dados para verificar a existência de padrões distintos de interação universidade-empresa para empresas nacionais e multinacionais. As conclusões do trabalho sugerem que não existem diferenças significativas entre os padrões de cooperação de empresas nacionais e empresas multinacionais com universidades/IPPs brasileiros.
\end{abstract}

Palavras-chave: empresas multinacionais; empresas nacionais; interação universidade/empresa.

\footnotetext{
1 Graduado em Ciências Econômicas pela Universidade Federal de Minas Gerais (UFMG). Pesquisador do Cedeplar/UFMG. E-mail: fabioi@cedeplar.ufmg.br

2 Doutorando em Economia pelo Cedeplar/UFMG. Bolsista do CNPq. E-mail: ulisses@cedeplar.ufmg.br

3 Mestre em Política Científica e Tecnológica pela Universidade Estadual de Campinas (Unicamp). Pesquisadora do Cedeplar/UFMG. E-mail: vparreiras@cedeplar.ufmg.br

4 Mestranda em Economia na Universidade Federal de Viçosa (UFV). E-mail: prigomesc@yahoo.com.br

5 Graduada em Ciências Econômicas pela Universiade Federal de Minas Gerais (UFMG). Pesquisadora do Cedeplar/UFMG. E-mail: luizamf@hotmail.com

6 Doutora em Economia pela Unicamp. Pesquisadora do IPEA. E-mail: fernanda.denegri@ipea.gov.br
} 


\title{
The Brazilian industry \& university/public research institutes's interaction: comparative relashionship evidence between domestic and multinational companies
}

\begin{abstract}
The aim of this paper is to investigate the interaction patterns of national and multinational enterprises located in Brazil with universities/ public research institutes (PRIs) in the country. To this end, we used a database involving 319 questionnaires answered by those responsible for the activities of research and development $(R \& D)$ and/or by interactions with universities and PRIs in enterprises located in Brazil, which interacted with research groups of Brazilian universities or PRIs in 2004. Such data were evaluated through a descriptive analysis to verify the existence of distinct patterns of university-industry interactions concerning national and multinational enterprises. The conclusions of the study suggest that there are not significant differences between the cooperation patterns of national and multinational companies with Brazilian universities/PRIs.
\end{abstract}

Key-words: multinational enterprises; national enterprises; universityindustry interactions.

JEL: L29; O10; O30.

Introdução

A literatura sobre empresas multinacionais (EMNs) salienta que a inovação é um dos segmentos menos internacionalizados dentro de sua estrutura, sendo uma das últimas atividades a serem deslocadas para outros países, posteriormente a atividades como a produção e o marketing (UNCTAD, 2005). No que diz respeito à natureza das atividades de Pesquisa e Desenvolvimento (P\&D) conduzidas nas subsidiárias, observa-se que uma grande parte dessas está associada à adaptação e modificação dos produtos e processos às necessidades dos mercados locais (OCDE, 1998, Dunning, 1992; UNCTAD, 2005; De Negri, 2007). Entretanto, algumas evidências sugerem uma crescente importância das atividades de P\&D das subsidiárias de EMNs com o objetivo de absorção e aquisição de transbordamentos (spill-overs) tecnológicos, tanto da base local de conhecimento quanto de firmas específicas (CRISCUOLO et al., 2005). Estratégias de busca tecnológica e de racionalização das atividades de P\&D ao redor do mundo - buscando economias de escala e de escopo - têm sido cada vez mais relevantes na decisão das EMNs de investir em P\&D em outros países. A internacionalização das atividades de P\&D destas empresas tem resultado no aumento das responsabilidades e capacidades tecnológicas das subsidiárias. 
No caso brasileiro, dados da Pesquisa de Inovação Tecnológica (PINTEC) e do Instituto Brasileiro de Geografia e Estatística (IBGE) de 2005 mostram que, no âmbito das empresas estrangeiras, o gasto com o P\&D interno das 631 empresas que revelaram realizar a atividade foi de aproximadamente $\mathrm{R} \$ 3,7$ bilhões, sendo o principal setor a indústria de fabricação de produtos químicos. A realização de P\&D externo foi verificada para 231 empresas estrangeiras, principalmente na indústria de transformação, com destaque também para a fabricação de produtos químicos. Em termos relativos, verifica-se que cerca de $50 \%$ dos investimentos privados em $\mathrm{P} \& \mathrm{D}$ realizados pela indústria brasileira em 2005 foram feitos por subsidiárias de empresas estrangeiras presentes no Brasil (DE NEGRI, 2007).Uma vez que o número de subsidiárias de empresas estrangeiras responsável por essa parcela da $P \& D$ no país é bem menor que o de empresas nacionais, é possível dizer que há uma maior intensidade deste gastos por partes das empresas de origem externa. Estes dados mostram a existência de um esforço das EMNs em desenvolver tecnologia no país, indicando um possível maior dinamismo destas em termos da busca pela inovação. Contudo, resta saber se tais esforços compreendem a cooperação com atores constituintes do sistema de inovação brasileiro.

Frente a este contexto, o objetivo deste trabalho é investigar os padrões de interação de empresas nacionais (ENs) e empresas multinacionais (EMNs) localizadas no Brasil com universidades/institutos públicos de pesquisa (IPPs) presentes no país. Para tanto, utilizou-se uma base de dados obtida por meio de um survey que resultou em 319 questionários respondidos no ano de 2009.

O texto está organizado em cinco seções, além desta introdução. A segunda seção apresenta uma revisão da literatura discutindo primeiramente aspectos das EMNs, com foco nas suas interações com universidades e IPPs em sistemas de inovação maduros e imaturos. A revisão da literatura ainda aborda o tema da internacionalização da P\&D e a atuação tecnológica das EMNs no Brasil. A terceira seção apresenta uma contextualização da interação entre universidades/IPPs e empresas no Brasil por origem do capital das empresas, elaborada com base nos dados do Diretório dos Grupos de Pesquisa no Brasil (DGP) do Conselho Nacional de Desenvolvimento Científico e Tecnológico (CNPq). A metodologia, apresentada na quarta seção, explica a pesquisa que originou o survey do qual foram extraídos os dados utilizados. A quinta seção investiga a existência ou não de padrões distintos de interação com universidades e IPPs para ENs e EMNs, a partir dos resultados obtidos por meio da análise descritiva dos dados do survey de empresas. Finalmente, na última seção são feitas as considerações finais acerca do trabalho. 


\section{Empresas multinacionais, sistemas de inovação imaturos e internacionalização da P\&D}

\subsection{EMNs, sistemas nacionais de inovação e a interação universidade-empresa}

As EMNs exercem um papel importante em meio aos sistemas nacionais de inovação, dado que muitas vezes contribuem com a introdução de novos conhecimentos e tecnologias nas economias nas quais se inserem. As atividades tecnológicas das filiais de EMNs contribuem para o fortalecimento da inovação local, direta e indiretamente, por meio do estímulo à competição e de atividades de assistência prestadas às empresas parceiras (Cantwell, 1994). Esta seria uma das razões pelas quais economias periféricas visam à entrada de firmas estrangeiras em seu território como tentativa de se conectarem aos desenvolvimentos tecnológicos internacionais. Isso ocorre, pois, as EMNs geralmente estão entre as líderes internacionais em desenvolvimento tecnológico (UNCTAD, 2005).

Contudo, o impacto das EMNs na criação e na organização eficiente de capacidades físicas e humanas pode ser diferente, de acordo com os home e host countries ${ }^{7}$ envolvidos neste processo. A maioria dos grandes países industriais, em um estágio similar de desenvolvimento econômico e produzindo uma variedade similar de produtos e serviços, se empenham em transações tecnológicas cross-border para sustentar ou avançar sua posição competitiva, um em relação ao outro. No caso de países em diferentes estágios de desenvolvimento e produzindo diferentes bens e serviços, os tipos de capacidades tecnológicas que as EMNs estão propensas a produzir e usar tendem a ser complementares (Dunning, 1992). De fato, a maioria da P\&D realizada em países menos desenvolvidos é designada especificamente às suas necessidades locais ou de países adjacentes, e ganham a forma de adaptação de produtos e processos às condições locais (OCDE, 1998; Dunning, 1992; UNCTAD, 2005; De Negri, 2007). Deste modo, observa-se que os esforços tecnológicos das EMNs em economias menos desenvolvidas seriam menos efetivos que em economias centrais.

Já a interação entre universidades e IPPs e empresas, tanto ENs quanto EMNs, remete ao grau de desenvolvimento do Sistema Nacional de Inovação (SNI). Este é constituído por um conjunto de agentes, como universidades, institutos de pesquisa, sistema financeiro e empresas, entre outros, que por meio de suas interações fomentam o processo de inovação tecnológica. Nesse contexto, é atribuída importância decisiva à interação entre a produção científica e a tecnológica, demonstrando que as universidades e os IPPs envolvidos na produção de ciência básica e aplicada são integrantes fundamentais do NSI. Isto ocorre, pois tais instituições agem de modo a incentivar e complementar as atividades inovativas desenvolvidas pela indústria.

7 Home country é o país de origem da empresa multinacional e host country é o país anfitrião, que recebe uma filial da empresa multinacional. 
Na esfera científica, as universidades e os IPPs desempenham um papel central na criação e na difusão do conhecimento através de funções tradicionais, como o ensino e a pesquisa básica. Desta forma, criam e renovam o estoque de conhecimento existente no país em que atuam, desempenhando um papel extremamente importante no desenvolvimento tecnológico, seja na formação e treinamento de engenheiros e cientistas industriais, seja como fonte de resultados de pesquisa e técnicas relevantes para o avanço técnico na indústria (Nelson; Rosenberg, 1993). No caso de economias periféricas, conforme Albuquerque (1999), a infra-estrutura científica pode atuar como uma "antena" para a identificação das oportunidades tecnológicas, conectando tais SNIs aos fluxos científicos e tecnológicos internacionais. Deste modo, a principal contribuição do sistema universitário a economias menos desenvolvidas residiria no suporte oferecido ao processo de catching up.

Na esfera tecnológica, as firmas geralmente não possuem todos os recursos necessários para inovar e, assim, necessitam adquirir conhecimento de fontes externas, como universidades e IPPs. A literatura a este respeito salienta a existência de algumas características estruturais relativas à capacidade de absorção destes agentes e à sua procura por estreitas interações com parceiros externos. Desta forma, enfatiza que para que a transferência de conhecimentos ocorra, a firma necessita deter alguma capacidade de absorção. Por apresentarem tal característica, as grandes corporações, como as EMNs, por seu poderio econômico e escala de atuação, geralmente estão entre os principais agentes inovadores no contexto internacional (UNCTAD, 2005).

No que tange às conexões entre estas duas esferas, como ressaltado por Albuquerque et al. (2008), é preciso fazer uma distinção no que diz respeito à interação universidade-empresa em SNIs maduros e imaturos. A literatura sobre SNIs maduros (Albuquerque, 1999) demonstra que nos países desenvolvidos é possível identificar padrões de interação entre as dimensões científica e tecnológica muito mais complexos do que o processo linear no qual a pesquisa básica dá origem a uma linha de pesquisa aplicada, a qual, por sua vez, gera uma inovação que resulta em lucro econômico (Narin et al., 1997). Fluxos de informação e de conhecimento ocorrem em ambas as direções, em relações interativas bidirecionais que promovem círculos virtuosos na produção e na difusão de conhecimento. Por um lado, universidades e IPPs produzem conhecimento que é absorvido pelo setor produtivo, conforme demonstrado por Narin et al. (1997), Klevorick et al. (1995) e Cohen et al. (2002). Por outro lado, as empresas acumulam conhecimento tecnológico que pode fornecer subsídio para a elaboração científica, conforme descrito por Rosenberg (1992). Na maioria dos países que apresentam um SNI maduro, faz-se presente, portanto, uma complementaridade entre a produção da pesquisa básica e a demanda da produção das firmas, além de canais mais fortes de relacionamento entre as partes, características essas que facilitam a comunicação entre elas (Albuquerque et al., 2008; Narin et al., 1997). 
SILVA NETO, F. C. C. et al. A interação universidades/institutos públicos de pesquisa...

As peculiaridades dos SNIs imaturos, como o brasileiro, quais sejam, a existência de "conexões parciais" entre a infra-estrutura científica e as atividades tecnológicas, limitam a interação entre os seus diversos elementos constituintes (Albuquerque, 1999; 2003). Isto porque o fluxo de conhecimento entre ciência e tecnologia fica restrito a um número reduzido de conexões ou interações que apresentam, nos casos bem sucedidos, um caráter localizado. (Rapini 2007; Albuquerque et al., 2008; Suzigan; Albuquerque, 2008).

\subsection{Processo de internacionalização da P\&D e a atuação tecnológica das EMNs no Brasil}

É fato que as atividades de P\&D das firmas estão cada vez mais internacionalizadas, embora permaneçam restritas a um pequeno número de países (Calrsson,2006; UNCTAD, 2005). Na visão de autores como Patel e Vega (1999), as atividades de P\&D das EMNs, apesar de serem cada vez mais realizadas fora do home country, ainda estão muito limitadas aos países desenvolvidos, mais especificamente aos países da tríade, Estados Unidos, Japão e Europa. No caso dos países em desenvolvimento, as atividades de P\&D destas firmas quase sempre estão ligadas à adaptação de produtos e processos aos mercados locais (Pearce, 1999; UNCTAD 2005).

Esse processo de descentralização das atividades tecnológicas está muito relacionado às estratégias atuais das EMNs, ligadas à necessidade de proteger, diversificar e complementar suas vantagens tecnológicas, assim como adquirir outras (Cantwell, 1994). Segundo Pearce (1999), as motivações para a descentralização da P\&D pelas EMNs englobam a reestruturação das subsidiárias de forma a apoiar a atuação competitiva da corporação, e ainda a emergência de competências tecnológicas distintas em um maior número de países. Entre estes, encontram-se alguns países em desenvolvimento, onde além da adaptação de produtos, as atividades de P\&D das EMNs passaram a englobar práticas mais complexas semelhantes a trabalhos desenvolvidos nos países avançados. No entanto, trata-se ainda de um número restrito de países em desenvolvimento e economias em transição que estão participando efetivamente do processo de internacionalização da $\mathrm{P} \& \mathrm{D}$, com destaque para os países asiáticos, como Coréia do Sul, Singapura, Taiwan (China), Hong Kong (China) e Malásia (OECD, 2008).

No que tange à caracterização das atividades tecnológicos das subsidiárias de EMNs no Brasil, estudos baseados nos surveys de inovação PINTEC/IBGE (Queiroz; Carvalho, 2005; Araujo, 2005; Ruiz; Bhawan, 2010) e Pesquisa da Atividade Econômica Paulista-PAEP/SEADE (Quadros et al., 2001; Costa; Queiroz, 2002;) mostram que estas empresas, tomadas em conjunto, são bastante ativas em P\&D e respondem por parcela significativa do esforço tecnológico das empresas instaladas no país. 
Dados sobre patentes podem ser utilizados para os mesmos fins. Albuquerque (2000) e Biazzi e Albuquerque (2001) demonstram que essas empresas (capital estrangeiro) têm uma importante participação nas atividades tecnológicas brasileiras, medida pelas patentes domésticas (patentes concedidas a residentes) pelo Instituto Nacional de Propriedade Industrial (INPI) Entretanto, os autores identificam um expressivo desequilíbrio entre os estoques de patentes depositadas "de fora para dentro" (patentes de não-residentes depositadas no Brasil pelas empresas globais) e de patentes depositadas por suas subsidiárias, um indicativo de baixa internalização relativa das atividades tecnológicas.

A literatura que procura caracterizar as atividades tecnológicas desenvolvidas pelas filiais de EMNs locais, por meio de surveys de empresas e estudos de caso em profundidade, também identifica que é expressiva a representação de empresas que realizam algum tipo de atividade tecnológica no país. Entretanto, alguns autores defendem a posição de que parcela considerável das subsidiárias de EMNs no Brasil realiza primordialmente atividades tecnológicas de Desenvolvimento (ou seja, o “D” da P\&D) (Dias, 2003; Consoni, 2004; Queiroz; Carvalho, 2005; Galina et al., 2005; Camillo et al., 2008; Paranhos; Hasenclever, 2009). Particularmente, Camillo et al. (2008) observam que, em algumas destas empresas, as atividades de desenvolvimento não são nem mesmo contínuas.

É importante observar que alguns autores (Queiroz; Carvalho, 2005; Camillo et al., 2008) argumentam que são raros os casos em que as funções de P\&D de uma subsidiária no país são diversificadas o suficiente para caracterizar a existência de atividades de pesquisa tecnológica interna à empresa. Contudo, outros trabalhos como Gomes et al. (2010) relativizam esta questão, demonstrando que a maioria das filiais pesquisadas realiza atividades tanto de pesquisa quanto de desenvolvimento no Brasil. Acrescente-se que Camillo et al. (2008) indicam que dentre as filiais de EMNs que realizam pesquisa tecnológica no Brasil, estas atividades são realizadas majoritariamente de forma esporádica.

Adicionalmente, a literatura demonstra que as atividades tecnológicas de subsidiárias atuantes no Brasil estão focadas primordialmente na adaptação de produtos e processos para os mercados locais e, em alguns casos, regionais (Quadros et al., 2001; Araujo, 2005; Queiroz; Quadros, 2005; Galina et al., 2005; Camillo et al., 2008; Strachman; Avellar, 2008). Assim, poderse-ia argumentar que é pouco expressiva a participação de subsidiárias que desenvolvem, no Brasil, pesquisa relevante para o desenvolvimento tecnológico global ou para a corporação no contexto global.

Cabe ressaltar, todavia, que estudos empíricos identificam subsidiárias no Brasil que acumularam capacidades tecnológicas relevantes, sendo que algumas delas possuem relevância no contexto global da corporação, considerando as competências acumuladas localmente. Subsidiárias de EMNs 
dos setores automobilístico (Dias, 2003; Consoni, 2004; Pereira, 2009), de equipamentos de telecomunicações (Galina et al., 2005) e dos segmentos de equipamentos mecânicos, elétricos e motores elétricos no setor de bens de capital (Strchman; Avellar, 2008) constituem os exemplos destacados pela literatura, seja pela (re)definição de mandatos tecnológicos por parte dos grupos aos quais pertencem, seja pela introdução ou ampliação de atividades tecnológicas já realizadas por estas subsidiárias. Por outro lado, constata-se que em certos setores amplamente dominados por EMNs no Brasil, como o farmacêutico, os esforços locais de P\&D são muito limitados, compreendendo essencialmente atividades de desenvolvimento referentes a testes clínicos (Camillo et al., 2008; Paranhos; Hasenclever, 2009).

Para os fins deste trabalho, é importante observar que há poucos estudos que investigam as relações de cooperação de filiais de EMNs instaladas no Brasil com universidades e IPPs. Posto isto, cabe salientar, primeiramente, que, de um modo geral, as empresas produtivas brasileiras cooperam muito pouco, particularmente com universidades e IPPs, diferentemente do que ocorre com as empresas dos países mais avançados (Cassiolato et al., 2005). Estes mesmos autores indicam que estas parcerias são extremamente reduzidas, mesmo no que se refere às firmas brasileiras que inovam e diferenciam produtos, as quais representam as empresas mais dinâmicas. É importante observar que Albuquerque et al. (2005), também com base nos dados da PINTEC 2000, demonstram que empresas com atividades de P\&D (interna e/ou externa) dão mais importância às universidades como fonte de informação para suas atividades inovativas do que as que não realizam atividades de P\&D. Quadros et al. (2001) e Queiroz e Carvalho (2005) desenvolvem uma argumentação convergente, sugerindo que a fragilidade do relacionamento das empresas industriais brasileiras com universidades e outras instituições de pesquisa é, em grande medida, determinada pelo baixo grau de formalização da atividade de P\&D nestas empresas

No conjunto da literatura que enfoca especificamente as relações de cooperação de subsidiárias de EMNs no Brasil com universidades e IPPs, cabe mencionar que Araujo (2005) identificou nos dados da edição 2000 da PINTEC um percentual menor de ENs que utilizaram alguma forma de cooperação para inovar em relação às EMNs instaladas no Brasil, inclusive com universidades e IPPs. Acrescente-se que depois de outra empresa do grupo localizada no exterior, provavelmente da matriz, ou da filial de um país com um SNI evoluído, destacam-se os clientes ou consumidores localizados no Brasil como parceiros para inovação para as EMNs, o que, segundo o autor, pode ser um indicativo de que provavelmente as EMNs desenvolvem produtos ou processos voltados muito mais para adaptação às condições locais. Já Costa e Queiroz (2002), com base nos dados da PAEP 1996, não encontraram diferenças significativas entre as filiais de EMNs e as ENs quanto às suas capacidades tecnológicas acumuladas de monitoramento e interação com outros agentes locais, particularmente com o sistema de C\&T. Esta posição é 
convergente com a de Ruiz e Bahwan (2010) em estudo embasado na PINTEC 2003 que investiga o aprendizado que resulta da interação da empresa com o exterior, das relações com fornecedores e usuários na cadeia produtiva e da cooperação (learning by interacting) e o aprendizado realizado sobre os avanços na ciência e tecnologia por meio da contratação de atividades de pesquisa de outras empresas ou de universidades e IPPs (learning by hiring) por parte de EMNs e ENs. As autoras encontram diferenças muito pequenas entre estes dois tipos de firmas no interior de cada setor de atividade econômica, prevalecendo a constatação dos imperativos setoriais na definição dos processos de aprendizagem.

Camillo et al. (2008), por sua vez, apresentam evidências de diferentes subsidiárias que não desenvolvem atividades de P\&D contínuas e formalizadas no país e que contratam esporadicamente universidades e IPPs brasileiros para a prestação de serviços tecnológicos relacionados à adaptação local de produto ou processo e para desenvolvimento(s) específico(s), de acordo com demandas específicas de mercado. A ausência de vínculos de longo prazo com universidades e IPPs brasileiros é, segundo os autores, uma situação comum às subsidiárias que focam meramente as atividades tecnológicas de desenvolvimento no país, sendo também verificada nos casos das empresas que realizam esforços de desenvolvimento de forma contínua e de pesquisa de maneira esporádica.

\section{Interação universidades/IPPs e empresas no Brasil, segundo o Diretório dos Grupos de Pesquisa (DGP/CNPq)}

Esta seção apresenta uma discussão introdutória da interação universidade/ IPP-empresa no Brasil, por origem do capital das empresas parceiras, a partir dos dados disponíveis na base do DGP/CNPq (Censo 2008) ${ }^{8}$. Na análise que segue, essas empresas estão estratificadas de acordo com a propriedade do seu capital.

A Tabela 1 apresenta o número de grupos de pesquisa interativos bem como o número de empresas interativas, sendo calculada a partir daí a densidade da interação destes agentes. Observa-se que há uma maior densidade da interação para as EMNs em comparação às ENs e ao conjunto de empresas interativas localizadas no país. Tal resultado aponta que, apesar de serem minoria na base do DGP/CNPq, as EMNs são, proporcionalmente, mais interativas que as ENs. 
SILVA NETO, F. C. C. et al. A interação universidades/institutos públicos de pesquisa...

TABELA 1. EMPRESAS E GRUPOS DE PESQUISA QUE INTERAGEM, SEGUNDO A ORIGEM DO CAPITAL DA EMPRESA. BRASIL - 2008

\begin{tabular}{lcc|c}
\hline $\begin{array}{c}\text { Origem do } \\
\text { capital }\end{array}$ & $\begin{array}{c}\text { Número de } \\
\text { Grupos (A) }\end{array}$ & $\begin{array}{c}\text { Número de } \\
\text { empresas (B) }\end{array}$ & $\begin{array}{c}\text { Densidade de } \\
\text { interação (A/B) }\end{array}$ \\
\hline Nacional & 1788 & 2280 & 0,78 \\
Multinacional & 349 & 325 & 1,07 \\
Total & 2137 & 2605 & 0,82 \\
\hline
\end{tabular}

FONTE: DGP/ CNPq, Censo 2008.

Pode-se verificar ainda, por meio da Tabela 2, como os padrões de interação com grupos de pesquisa envolvendo ENs e EMNs podem variar de acordo com as áreas de conhecimento dos grupos. Os dados sugerem que há um padrão similar de interação entre os grupos de pesquisa e as ENs e EMNs: as interações com os grupos da área "engenharias" são as mais recorrentes, tanto para ENs quanto para EMNs, embora sejam maiores no segundo caso. Cabe salientar que as demais diferenças observadas pela Tabela 2, no que diz respeito às EMNs e ENs, não são tão significativas como no caso da área de conhecimento "engenharias".

TABELA 2. GRUPOS INTERATIVOS COM EMPRESAS, SEGUNDO A ORIGEM DO CAPITAL DA EMPRESA. BRASIL - 2008

\begin{tabular}{lccc}
\hline \multicolumn{1}{c}{ Origem do capital da empresa } & Grupos (A) & $\%$ \\
\hline Nacional & 1788 & 100,00 \\
\hline Engenharias & 605 & 33,84 \\
Ciências Agrárias & 364 & 20,36 \\
Ciências exatas e da Terra & 298 & 16,67 \\
Ciências Biológicas & 182 & 10,18 \\
Ciências da saúde & 164 & 9,17 \\
Ciências Sociais Aplicadas & 103 & 5,76 \\
Ciências Humanas & 60 & 3,36 \\
Linguística, letras e artes & 12 & 0,67 \\
\hline Multinacional & 349 & 100,00 \\
\hline Engenharias & 159 & 45,56 \\
Ciências Agrárias & 78 & 22,35 \\
Ciências exatas e da Terra & 49 & 14,04 \\
Ciências Biológicas & 26 & 7,45 \\
Ciências da saúde & 24 & 6,88 \\
Ciências Sociais Aplicadas & 7 & 2,01 \\
Ciências Humanas & 6 & 1,72 \\
Linguística, letras e artes & 0 & 0,00 \\
\hline Total & 2.137 & 100,00 \\
\hline
\end{tabular}

FONTE: DGP/ CNPq, Censo 2008. 
No que tange aos tipos de relacionamento entre ENs e EMNs e grupos de pesquisa, os Gráficos 1 e 2 mostram que a principal forma de relacionamento entre estas instituições é a pesquisa científica. Os tipos de relacionamento "pesquisa científica sem consideração de uso imediato" e "pesquisa científica com considerações de uso imediato", conjuntamente, representam $43 \%$ dos relacionamentos com grupos de pesquisa, tanto para EMNs quanto para ENs. Os dados do CNPq apontam para o fato de que há uma maior recorrência, nos dois casos, de pesquisa com considerações de uso imediato em relação à pesquisa sem considerações de uso imediato. Assim sendo, nos dois conjuntos de empresas há preferência pela interação que traga resultados no curto prazo.

Observa-se, ainda, que as EMNs e ENs não apresentam grandes divergências no que diz respeito aos demais tipos de relacionamento observados. Constatase que, proporcionalmente, a transferência de tecnologia aparece com maior freqüência no caso das ENs. Já o fornecimento de insumos materiais é mais recorrente no caso das EMNs. Não obstante tais diferenças, a ordem de importância dos tipos de relacionamento entre as partes é praticamente a mesma, para os dois tipos de empresas.

GRÁFICO 1. TIPOS DE RELACIONAMENTO DE GRUPOS DE PESQUISA E ENS (\%). BRASIL - 2008

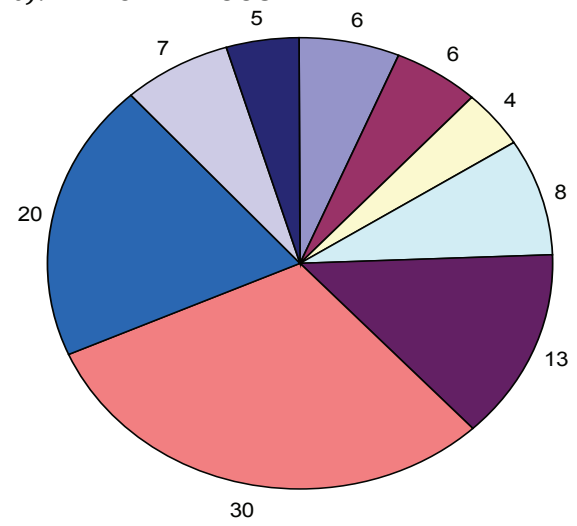

$\square$ Atividades de consultoria técnica

$\square$ Atividades de engenharia nãorotineira*

$\square$ Desenvolvimento de software.*

$\square$ Fornecimento de insumos materiais*

$\square$ Pesquisa científica sem considerações de uso imediato.

$\square$ Pesquisa científica com considerações de uso imediato.

$\square$ Transferências de tecnologia*

$\square$ Treinamento de pessoal, incluindo cursos e treinamento "em serviço". *

$\square$ Outros tipos de relacionamentos

NOTA: * São relacionamentos bilaterais.

FONTE: DGP/ CNPq, Censo 2008. 
GRÁFICO 2. TIPOS DE RELACIONAMENTO DE GRUPOS DE PESQUISA E EMNS (\%), BRASIL - 2008

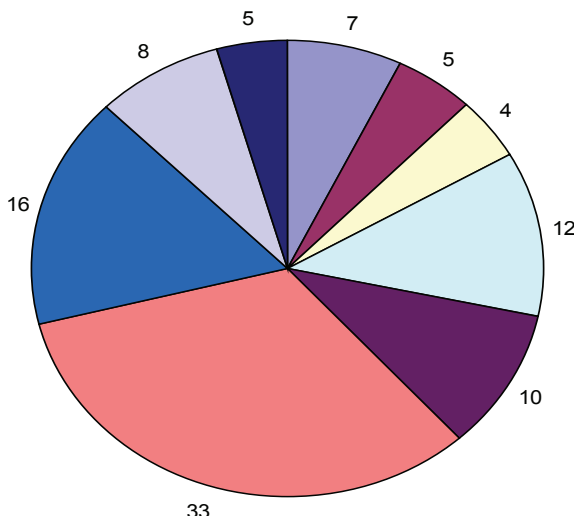

33

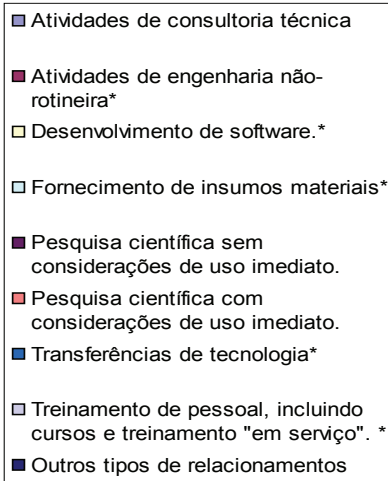

NOTA: * São relacionamentos bilaterais.

FONTE: DGP/ CNPq, Censo 2008.

\section{Metodologia}

Este artigo apresentará adiante os resultados de parte de uma pesquisa ${ }^{9}$ realizada com empresas, instituições de pesquisa e departamentos de P\&D localizados no Brasil para a análise da dimensão tecnológica do país. No âmbito desta pesquisa, foi enviado um questionário a cada uma das 1.622 empresas localizadas no Brasil que interagiam com universidades/IPPs do país, conforme informações captadas pelo Censo de 2004 do DGP do CNPq. Nesse sentido, foi feito um levantamento dos grupos de pesquisa do Brasil cadastrados no DGP, cujo líder declarou algum relacionamento com o setor produtivo no Censo acima mencionado. Conforme metodologia proposta por Rapini (2004), as interações dos grupos de pesquisa do Diretório do CNPq podem ser utilizadas como proxy da interatividade de firmas e universidades. Deste modo, as empresas mencionadas pelos líderes dos grupos de pesquisa constituíram o foco desta investigação.

9 Para realização do survey, foram utilizados recursos de quatro projetos: Edital Universal CNPq "Interações de Universidades e Institutos de Pesquisa com Empresas no Brasil" (Processo: 478994/2006). IDRC - "Interactions between universities and firms: searching for paths to support the changing role of universities in Latin America". Fapesp - Projeto Temático "Interações de Universidades/Instituições de Pesquisa com Empresas Industriais no Brasil”. Processo 2006/58878-8, vigência entre 01/12/2007 e 30/11/2011. Fapemig - "Oportunidades Ao Desenvolvimento Sócio-Econômico e Desafios da Ciência, da Tecnologia e da Inovação em Minas Gerais” (CEX-1735/07). 
Das 22 questões presentes no questionário enviado aos responsáveis nas empresas pelas atividades de P\&D e/ou pelas interações com universidades/ IPPs, 5 são analisadas neste artigo. São elas: questão 1, referente às atividades inovativas e de $\mathrm{P} \& \mathrm{D}$ das empresas; questões 11 e 12, que dizem respeito às fontes externas de informação e conhecimento utilizadas para as atividades inovativas da empresa; questão 15, relacionada às razões da colaboração com universidades/IPPs; e a questão 17, referente à avaliação por parte das empresas dos projetos em colaboração com as universidades e IPPs.

Para as análises descritas na seção 5 deste trabalho, as instituições que responderam ao questionário, foram classificadas em empresas e outros tipos de instituição, com base na natureza jurídica declarada por essa empresa na Relação Anual de Informações Sociais (RAIS), do IBGE. Para isso, a base de dados originada do survey foi ligada à base de dados da RAIS, por meio do CNPJ (Cadastro Nacional da Pessoa Jurídica) da empresa/instituição. Foram classificadas como empresas todas as instituições que declararam, na RAIS, sua natureza jurídica como sendo "empresa pública" ou "empresa privada" e que não pertençam aos seguintes setores de atividade: i) educação; ii) atividades associativas e; iii) administração pública. Dessa forma, do universo de 319 questionários respondidos restaram, na base tratada, 251 empresas. As informações referentes a estas empresas, por sua vez, foram cruzadas com o Censo de Capitais Estrangeiros, do Banco Central, de onde foi possível identificar a origem de capital da empresa, se estrangeira ou nacional. Constatou-se que, desta totalidade, estão presentes na base de dados da pesquisa 32 empresas multinacionais, o que corresponde a um percentual de $12,8 \%$ da amostra.

\section{Análise dos resultados}

Nesta seção reliza-se uma análise de algumas das principais características dos processos de cooperação entre universidade/IPPs e empresas no Brasil, sendo estas últimas diferenciadas em ENs e EMNs, a partir de informações do survey de empresas anteriormente descrito.

No que tange às classes de tamanho, as EMNs cobertas pela pesquisa distribuem-se da seguinte forma: 46,9\% delas são grandes empresas (500 ou mais empregados), 43,8\% de médio porte (entre 100 e 499 empregados) e apenas 9,4\% de pequeno porte (menos de 100 empregados). Já na composição das ENs que respondeu o survey as empresas distribuem-se de forma mais equilibrada entre as classes de tamanho: 33,3\% delas são grandes empresas; 28,8\% de médio porte e $36,5 \%$ de pequeno porte. A proporção da mão-de-obra empregada em P\&D é baixa em ambos os tipos de empresa (2,0\% no total), embora maior nas ENs $(2,1 \%)$ do que nas EMNs $(1,2 \%)$

Sobre a introdução de inovações, na tabela 3, é possível verificar que 92,4\% das 
empresas entrevistadas (ou 92,2\% das ENs e 93,8\% das EMNs) introduziram inovações de produto nos últimos 3 anos e 94,02\% (ou 93,2\% das ENs e 100\% das EMNs) introduziram inovações de processo. Estes dados sugerem que há uma associação entre interação com a infra-estrutura científica nacional e inovação nas empresas entrevistadas, ou seja, que as empresas que possuem projetos conjuntos com universidades e IPPs são inovadoras. É notável também o fato de que todas as EMNs entrevistadas afirmaram ter realizado algum tipo de inovação nos últimos três anos.

Além disso, nota-se que as ENs e EMNs realizaram inovações de produtos considerados novos para o país e para o mundo na mesma proporção, embora as empresas de capital estrangeiro tenham realizado significativamente mais inovações de processo consideradas novos para o país e para o mundo do que as empresas de capital nacional - a proporção aproxima-se do dobro. Há nesse resultado algumas pistas que indicam, considerando a literatura já exposta, que ambos os tipos de empresas inovam em produtos na mesma proporção, seja para conquistar mercados, seja para adaptar produtos ao mercado brasileiro, no caso das EMNs. Entretanto, a disparidade de inovações de processo das EMNs em relação às ENs pode indicar que as primeiras inovam no processo produtivo dentro do contexto de adaptação deste às unidades locais, ao trazer o conhecimento do exterior para estas. E, nessa transferência, há tecnologias que podem ser consideradas novas para o país. A taxa de inovações das unidades locais de EMNs consideradas novas para o mundo - 18,3\% para produto e $15,6 \%$ para processo - é importante para medir em que grau a subsidiária nacional inova para o mercado global.

TABELA 3. EMPRESAS QUE DECLARARAM TEREM, OU NÃO, INTRODUZIDO ALGUMA INOVAÇÃO NOS ÚLTIMOS 3 ANOS, BRASIL - 2009

\begin{tabular}{lcc}
\hline \multicolumn{1}{c}{ Produtos } & ENs (\%) & EMNs (\%) \\
\hline Sem inovação & 7,8 & 6,3 \\
Inovação introduzida & 92,2 & 93,8 \\
\hline Novo para o país & 43,4 & 43,8 \\
Novo para o mundo & 18,3 & 18,8 \\
\hline \multicolumn{1}{c}{ Processos } & ENs (\%) & EMNs (\%) \\
\hline Sem inovação & 6,8 & - \\
Inovação introduzida & 93,2 & 100,0 \\
\hline Novo para o país & 24,2 & 40,6 \\
Novo para o mundo & 8,7 & 15,6 \\
\hline
\end{tabular}

NOTA: * As empresas poderiam responder mais de uma alternativa; ** Do total de 251 empresas - 219 nacionais e 32 multinacionais.

FONTE: Pesquisa de Campo BR Survey, 2009. 
Também foi contemplada a importância atribuída pelas empresas às fontes de informações de universidades e IPPs no desenvolvimento de suas atividades inovativas. Os dados da tabela 4 não mostram diferenças significativas entre a importância atribuída por ENs e EMNs às informações provenientes dessas instituições, uma vez que os percentuais de grande importância atribuídos às fontes de informação não diferiram muito. Entretanto, em relação às fontes de informação ligadas às universidades, pode-se notar duas exceções a respeito da maior importância atribuída ao recrutamento de pessoal graduado e pósgraduado e à troca informal de informações por parte das ENs, e da maior importância considerada à pesquisa encomendada a universidades e IPPs e realizada em conjunto, por parte das $\mathrm{EMNs}^{10}$.

A característica de complementaridade da pesquisa por parte da EMN se mostra ainda mais evidente ao analisar a importância das fontes de informação de IPPs. Na tabela 4, é possível verificar que as fontes de informação mais importantes para as EMNs são as pesquisas realizadas em conjunto e encomendadas aos IPPs, enquanto para as ENs são as publicações e conferências públicas/encontros.

Outro aspecto igualmente relevante refere-se à diferença da importância das trocas informais de informações para as ENs e as EMNs. As primeiras consideram as trocas informais muito mais importantes que as últimas, tanto quando a fonte é proveniente de universidades quanto de IPPs.

10 Entretanto, é necessário salientar que, em levantamento da importância das fontes de informação para as atividades inovativas de todas as empresas realizado neste survey, constata-se que a maior parte do conhecimento utilizado para as suas atividades inovativas não é proveniente de universidades e IPPs, mas de suas próprias linhas de produção. De fato, a linha de produção da empresa foi apontada como a fonte de informação mais importante, tanto para sugerir novos projetos como para completar os já existentes. As universidades foram consideradas como a terceira fonte mais importante para sugerir novos projetos e a segunda mais importante para completar os projetos já existentes. Já os IPPs aparecem como a terceira fonte mais importante para completar os projetos já existentes das empresas, embora, para sugerir novos projetos, apareçam apenas em sétimo lugar. 
SILVA NETO, F. C. C. et al. A interação universidades/institutos públicos de pesquisa...

TABELA 4. IMPORTÂNCIA DAS FONTES DE INFORMAÇÃO DE UNIVERSIDADES/IPPS PARA AS ATIVIDADES INOVATIVAS DAS EMPRESAS.

BRASIL, 2009

\begin{tabular}{|c|c|c|c|c|}
\hline \multirow[b]{2}{*}{$\begin{array}{l}\text { Fontes de Informação/ } \\
\text { Importância da fonte de } \\
\text { informação }\end{array}$} & \multicolumn{2}{|c|}{ Universidades } & \multicolumn{2}{|c|}{ IPPs } \\
\hline & $\begin{array}{l}\text { \% Mod. } \\
\text { ou Muito } \\
\text { importante } \\
(\mathrm{ENs})^{*}\end{array}$ & $\begin{array}{c}\text { \% Mod. } \\
\text { ou Muito } \\
\text { importante } \\
(\mathrm{EMNs})^{* *}\end{array}$ & $\begin{array}{l}\text { \% Mod. } \\
\text { ou muito } \\
\text { importante } \\
(\mathrm{ENs})^{*}\end{array}$ & $\begin{array}{l}\text { \% Mod. } \\
\text { ou muito } \\
\text { importante } \\
(\mathrm{EMNs})^{* *}\end{array}$ \\
\hline Patentes & 35,2 & 37,5 & 37,6 & 43,8 \\
\hline Publicações e relatórios & 72,1 & 71,9 & 61,9 & 56,3 \\
\hline $\begin{array}{l}\text { Conferências públicas e } \\
\text { encontros }\end{array}$ & 63,0 & 62,5 & 58,3 & 56,3 \\
\hline $\begin{array}{l}\text { Troca informal } \\
\text { de informações }\end{array}$ & 66,7 & 53,1 & 57,3 & 43,8 \\
\hline $\begin{array}{l}\text { Pessoal contratado com } \\
\text { graduação ou } \\
\text { pós-graduação }\end{array}$ & 67,1 & 56,3 & 49,5 & 50,0 \\
\hline Tecnologia licenciada & 33,8 & 31,3 & 34,4 & 34,4 \\
\hline $\begin{array}{l}\text { Consultoria com pesquisa- } \\
\text { dores individuais }\end{array}$ & 50,7 & 56,3 & 43,6 & 46,9 \\
\hline $\begin{array}{l}\text { Pesquisa encomendada à } \\
\text { universidade }\end{array}$ & 52,5 & 62,5 & 51,8 & 59,4 \\
\hline $\begin{array}{l}\text { Pesquisa realizada em con- } \\
\text { junto com a universidade }\end{array}$ & 67,1 & 75,0 & 56,9 & 68,8 \\
\hline $\begin{array}{l}\text { Participação em redes que } \\
\text { envolvam a universidade }\end{array}$ & 51,1 & 46,9 & 46,3 & 43,8 \\
\hline $\begin{array}{l}\text { Intercâmbio temporário } \\
\text { de pessoal }\end{array}$ & 37,0 & 28,1 & 30,7 & 28,1 \\
\hline Incubadoras & 22,8 & 28,1 & 21,6 & 28,1 \\
\hline $\begin{array}{l}\text { Parques científicos } \\
\text { e tecnológicos }\end{array}$ & 36,5 & 40,6 & 32,6 & 37,5 \\
\hline $\begin{array}{l}\text { Empresa pertence } \\
\text { a universidade }\end{array}$ & 14,6 & 15,6 & 21,1 & 18,8 \\
\hline $\begin{array}{l}\text { Empresa é spin-off } \\
\text { de universidade }\end{array}$ & 16,0 & 18,8 & 15,6 & 15,6 \\
\hline
\end{tabular}

NOTA: * Com base em 218 respostas - 1 empresa não respondeu; ** Com base em 32 respostas. FONTE: Pesquisa de Campo, BR Survey, 2009. 
Ao visualizar as fontes de informação de universidades e IPPs, como um todo, cabe salientar que ao atribuir maior peso a publicações, relatórios e conferências, as ENs procuram a transferência de conhecimento por meios que não exijam maiores custos de transferência, ou seja, que não demandam maiores investimentos na aquisição de conhecimento para a inovação. Contudo, estas vias exigem capacitações internas à firma para a absorção do conhecimento por elas demandado. A pouca valorização de empresas pertencentes a universidades ou que sejam spin-offs delas como fontes de informação para as empresas avaliadas no survey reafirma esta observação. Spin-offs e empresas pertencentes a universidades nascem como fruto de desenvolvimentos tecnológicos das universidades Por isso, o acesso ao seu conhecimento tecnológico por empresas já estabelecidas no mercado demandaria maiores custos a estas, devendo observar questões legais relativas à transferência de tecnologia ou à sua compra.

Ainda no que tange às vias de absorção de conhecimento pelas empresas, o contexto que explica a importância que as EMNs atribuem aos próprios projetos de pesquisa como fonte de informação é distinto, pelo fato de que estas empresas buscam colaborações pontuais e complementares, tanto com as universidades quanto com os IPPs.

TABELA 5. RAZÕES DA COLABORAÇÃO DA EMPRESA COM UNIVERSIDADES E/OU IPPS. BRASIL - 2009

\begin{tabular}{|c|c|c|}
\hline Razões da colaboração /Importância & $\begin{array}{l}\text { Mod. ou Muito } \\
\text { importante (ENs } \\
\text { em \%) }\end{array}$ & $\begin{array}{l}\text { Mod. ou mui- } \\
\text { to importante } \\
\text { (EMNs em \%) }\end{array}$ \\
\hline Transferência de tecnologia da Universidade & 64,38 & 37,50 \\
\hline $\begin{array}{l}\text { Buscar conselhos de cunho tecnológico ou consul- } \\
\text { toria com pesquisadores e/ou professores para a } \\
\text { solução de problemas realcionados à produção }\end{array}$ & 61,64 & 59,38 \\
\hline $\begin{array}{l}\text { Aumentar a habilidade da empresa para encon- } \\
\text { trar e absorver informações tecnológicas }\end{array}$ & 58,90 & 53,13 \\
\hline $\begin{array}{l}\text { Conseguir informações sobre engenheiros ou } \\
\text { cientistas e/ou tendências de P\&D nas áreas } \\
\text { científicas }\end{array}$ & 49,77 & 31,25 \\
\hline $\begin{array}{l}\text { Contratar pesquisas complementares, necessárias } \\
\text { para as atividades inovativas da empresa, em } \\
\text { universidades e institutos, centros ou laboratórios } \\
\text { de pesquisa }\end{array}$ & 60,27 & 53,13 \\
\hline $\begin{array}{l}\text { Contratar pesquisas que a empresa não pode } \\
\text { realizar }\end{array}$ & 57,53 & 68,75 \\
\hline $\begin{array}{l}\text { Fazer, o mais cedo possível, contatos com estu- } \\
\text { dantes universitários de excelência para futuro } \\
\text { recrutamento }\end{array}$ & 39,73 & 21,88 \\
\hline
\end{tabular}


Utilizar recursos disponíveis nas universidades e laboratórios de pesquisa

Realizar testes necessários para produtos e processos da empresa

Receber ajuda no controle de qualidade
63,93

56,25

65,30

65,63

30,14

25,00

NOTA: * 1 empresa não respondeu a questão. 219 empresas foram consideradas domésticas e 32 multinacionais.

FONTE: Pesquisa de Campo, BR Survey, 2009

Na tabela 5 pode-se observar o objetivo específico e complementar da interação das EMNs com essas instituições, ao atribuírem maior importância às razões destinadas a resolver soluções pontuais na produção da empresa, quais sejam contratar pesquisas que a empresa não pode desenvolver e realizar testes para produtos e processos da empresa. Com relação às ENs, a atribuição de maior importância à realização de testes, seguida de transferência de tecnologia e utilização de recursos de infra-estrutura, pode indicar que há maior interesse na absorção e utilização de conhecimentos externos, já que estas empresas poderiam ter maior dificuldade para gerar novas tecnologias, ou mesmo dificuldade de investir nestes procedimentos.

Finalmente, cabe analisar o alcance dos objetivos esperados pelas empresas na colaboração com universidades e IPPs. A avaliação das interações realizadas até o momento é muito positiva, tanto por parte das ENs quanto das EMNs, como mostra a tabela 6 . Esse sucesso pode ser muito relevante para incentivar o aumento dos relacionamentos entre os grupos de pesquisa de universidades e IPPs e empresas no Brasil. Particularmente, a boa avaliação da interação das EMNs com as universidades e IPPs locais $(86,67 \%)$ é positiva no sentido de apontar que o conhecimento produzido nos centros científicos do país atende às demandas das empresas com padrão de qualidade internacional.

TABELA 6. AVALIAÇÃO DO SUCESSO NA COLABORAÇÃO DAS EMPRESAS COM UNIVERSIDADES E IPPS, BRASIL - 2009

\begin{tabular}{l|c|c}
\hline & $\begin{array}{c}\text { Respostas* }^{*} \\
\text { (ENs) }\end{array}$ & $\begin{array}{c}\text { Respostas }^{*} \\
\text { (EMNs) }\end{array}$ \\
\hline Sim, colaboração é ou foi bem sucedida & 182 & 26 \\
Não, a colaboração não é ou foi bem sucedida & 20 & 3 \\
\hline
\end{tabular}

NOTA: *20 empresas não responderam a questão; ** Do total de 251 empresas.

FONTE: Pesquisa de Campo. BR Survey, 2009. 
A literatura aponta uma tendência ao aumento da internacionalização das atividades de P\&D na estrutura organizacional das EMNs. Esta tendência poderia induzir à ampliação das interações entre essas empresas e os agentes integrantes dos NSIs de economias periféricas nos processos inovativos. Nesse sentido, este trabalho objetivou avaliar os padrões de interação entre universidades/IPPs e empresas no Brasil, focando nas diferenças entre empresas de origem nacional e estrangeira.

Os dados do DGP/CNPq permitem verificar que não há diferenciação significativa entre os padrões de interação de universidades/IPPs e ENs e EMNs no país, tanto no que diz respeito às áreas dos grupos de pesquisa com as quais essas empresas interagem, quanto aos tipos de relacionamentos declarados pelos líderes desses grupos de pesquisa. Os dados do $\mathrm{CNPq}$ apontam que há uma maior recorrência, nos dois casos, de pesquisa com considerações de uso imediato em relação à pesquisa sem considerações de uso imediato.

Adicionalmente, a análise descritiva dos dados do survey mostrou que, apesar de haver algumas diferenças nos padrões de cooperação de ENs e EMNs, de um modo geral, as interações dessas empresas com universidades e IPPs são bastante similares. A principal diferença está relacionada aos motivos e aos objetivos da colaboração. Destaca-se o fato das EMNs darem maior importância à contratação de pesquisas junto a universidades/IPPs, enquanto as ENs atribuem maior importância à transferência de tecnologia das universidades/IPPs e à utilização de recursos disponíveis nestas instituições.

Os dados também sugerem que a cooperação das EMNs com universidades/ IPPs poderia ser caracterizada pela busca do atendimento de uma necessidade específica e pontual da empresa com relação ao desenvolvimento e aprimoramento de seus produtos e processos. As ENs, por outro lado, atribuem maior importância a fatores que podem estar relacionados ao aprendizado direto provindo da cooperação, tais como a transferência de tecnologia das universidades/IPPs e a utilização de recursos disponíveis nestas instituições, citados anteriormente. Apesar dessas diferenciações, observou-se que a importância atribuída por ENs e EMNs às informações provenientes de universidades e IPPs, assim como o perfil da empresa ser inovativa, o grau de satisfação e o grau de importância das razões de cooperação, são muito semelhantes para ambas as empresas.

Sendo assim, de acordo com a análise descritiva dos dados do survey de empresas não foi possível observar grandes diferenças nos padrões interativos de ENs e EMNs com universidades e IPPs brasileiros. Os resultados sugerem que a origem do capital não é um fator determinante para a diferenciação dos padrões de interação de universidades e IPPs com empresas no SNI brasileiro. 


\section{Referências bibliográficas}

ALBUQUERQUE, E. (1999). "National systems of innovation and non-OCED countries: notes about a rudimentary and tentative 'tipology'." Brazilian Journal of Political Economy, 19; p. 35-52.

ALBUQUERQUE, E. (2000). "Patentes de empresas transnacionais e fluxos tecnológicos com o Brasil: observações iniciais a partir de estatísticas de patentes depositadas e concedidas pelo INPI." Texto para discussão 134. Belo Horizonte: UFMG/Cedeplar.

ALBUQUERQUE, E. (2003). "Immature systems of innovation: introductory notes about a comparison between South Africa, India, Mexico and Brazil based on science and technology statistics." First Globelics Conference.

ALBUQUERQUE, E.; SILVA, L. A.; PÓVOA, L. (2005). "Diferenciação intersetorial na interação entre empresas e universidades no Brasil." São Paulo em Perspectiva, 19(1); p. 95-104.

ALBUQUERQUE, E.; SUZIGAN, W.; CÁRIO, S.; FERNANDES, A. C.; SHIMA, W.; BRITTO, J.; BARCELOS, A.; RAPINI, M. (2008). "An investigation on the contribution of universities and research institutes for maturing the Brazilian innovation system: preliminary results." Fourth Globelics Conference.

ARAUJO, R. (2005). "Esforços tecnológicos de firmas transnacionais e domésticas.” In: DE NEGRI, J.; SALERNO, M. [orgs]. Inovações, Padrões Tecnológicos e Desempenho das Firmas Industriais Brasileiras. IPEA.

BIAZZI, E.; ALBUQUERQUE, E. (2001). "Transnational corporations and patenting activities in Brazil: data description and statistical tests about the relative internalization of technological activities." Revista de Economia Aplicada, 5(2); p. 407-431.

CALRSSON, B. (2006). "Internationalization of innovation systems: a survey of the literature." Research Policy, 35; p. 56-67.

CAMILLO, E.; GALINA, S.; CONSONI, F. L. (2008). "FDI in R\&D: what the MNCs subsidiaries are doing in Brazil." Anais do GLOBELICS 6th International Conference. URL [on-line]: http://globelics_ conference2008.xoc.uam.mx/papers/Edilaine_Camillo_FDI_in_R_D. pdf. Acesso em: 01 de dez. de 2010. 
CANTWELL, J. (1994). "Introduction: transnational corporations and innovatory activities.” In: DUNNING, J. H. (ed.). Transnational corporations and innovatory activities. London: Routledge.

CASSIOLATO, J. E.; BRITTO, J.; VARGAS, M. A. (2005). “Arranjos cooperativos e inovação na indústria brasileira." In: DE NEGRI, J.; SALERNO, M. S. [Orgs.]. Inovações, padrões tecnológicos e desempenho das firmas industriais brasileiras. Brasília: IPEA.

COHEN, W.; NELSON, R.; WALSH, J. (2002). "Links and impacts: the influence of public R\&D on industrial research." Management Science, 48(1); p. 1-23.

CONSONI, F. (2004). Da tropicalização ao projeto de veículos: um estudo das competências em desenvolvimento de produto nas montadoras de automóveis no Brasil. Campinas: Tese de Doutorado. Universidade Estadual de Campinas, Departamento de Política Científica e Tecnológica.

COSTA, I.; QUEIROZ, S. (2002). "Foreign direct investment and technological capabilities in brazilian industry." Research Policy, 31: 1.431-1.443.

CRISCUOLO, P.; NARULA, R.; VERSPAGEN, B. (2005). "Role of home and host country innovation systems in R\&D internalization: a patent citation analysis." Economics of Innovation and New Technology, 14(5); p. $417-433$.

DE NEGRI, F. (2007). Investimento direto e transferência de tecnologia: Argentina, Brasil e México. 2007. Campinas: Tese de Doutorado. Universidade Estadual de Campinas, Instituto de Economia.

DIAS, A. V. C. (2003). Projeto Mundial, Engenharia Brasileira: integração de subsidiárias no desenvolvimento de produtos globais da indústria automobilística. São Paulo: Tese de Doutorado. Escola Politécnica da Universidade de São Paulo (USP)

DUNNING, J. (1992). Multinational enterprises and the global economy. Wokingham, pp.331-348.

GALINA, S.; SBRAGIA, R.; PLONSKI, G. A. (2005). "R\&D in the telecom industry in Brazil: some indicators involving large transnational." Innovation: Management, Policy \& Practice, 7(2); p. 298-309. 
GOMES, R. et all. (2010). "Fatores de Atração de Atividades de Pesquisa e Desenvolvimento (P\&D): um survey das filiais de empresas multinacionais instaladas no Brasil." Anais do $38^{\circ}$ Encontro Nacional de Economia. URL [on-line]: <http://www.anpec.org.br/encontro_2010. htm\#TRABALHOS>. Acesso em: 10 de jan. de 2011.

INSTITUTO BRASILEIRO DE GEOGRAFIA E ESTATÍSTICA (2005). Pesquisa Industrial de Inovação Tecnológica. Rio de Janeiro: IBGE.

KLEVORICK, A.; LEVIN, R.; NELSON, R.; WINTER, S. (1995). "On the sources and significance of inter-industry differences in technological opportunities." Research Policy, 24(2); p. 185-205.

NARIN, F.; HAMILTON, K. S.; OLIVASTRO, D. (1997). "The increasing linkage between US technology and public science.” Research Policy, 26(3); p. 317-330.

NELSON, R.; ROSENBERG, N. (1993). "Technical innovation and national systems.” In: NELSON, R. (Ed.). National Innovation Systems: a comparative analysis. New York: Oxford University Press.

ORGANISATION FOR ECONOMIC CO-OPERATION AND DEVELOPMENT (1998). $L$ internalisation de la $R-D$ industrielle: strcutures et tendences. Paris: Organisation de Coopération et de Développement Écononomiques.

ORGANISATION FOR ECONOMIC CO-OPERATION AND DEVELOPMENT. (2008). Globalisation of industrial $R \& D$ : policies issues. 110p.

PARANHOS, J.; HASENCLEVER, L. (2009). "Industry-University interactions in the pharmaceutical system of innovation: Brazilian and international data." In: DRUID-DIMEAcademy Winter. Aalborg: DRUIDDIME. URL [on-line]: http://www2.druid.dk/conferences/viewpaper. php?id=4225\&cf=33. Acesso em: 14 de jan. de 2011.

PATEL, P.; VEGA, M. (1999). "Patterns of internationalization of corporate technology: location vs. home country advantages.” Research Policy, 28; p. 145-155.

PEARCE, R. D. (1999). “Decentralised R\&D and Strategic Competitiveness: globalized approaches to generation and use of technology in multinational enterprises (MNEs).” Research Policy, 28; p. 157-178.

PEREIRA, H. C. (2009). Global innovation networks: um estudo de caso. Belo Horizonte: Monografia de Graduação. Universidade Federal de Minas Gerais, Faculdade de Ciências Econômicas. 
QUADROS, R.; FRANCO, E.; FURTADO, A.; BERNARDES, R. (2001). "Technological Innovation in Brazilian Industry: An Assessment Based on the São Paulo Innovation Survey." Technological Forecasting and Social Change, 67; p. 203-219.

QUEIROZ, S.; CARVALHO, R. (2005). "Empresas multinacionais e inovação tecnológica no Brasil.” São Paulo em Perspectiva, 19(2); p. 51-59.

RAPINI, M. S. (2004). Interação Universidade-Indústria no Brasil: uma análise exploratória a partir do Diretório dos Grupos de Pesquisa do CNPq. Rio de Janeiro: Dissertação de Mestrado. Universidade Federal do Rio de Janeiro, Instituto de Economia.

RAPINI. M. (2007). "Interação Universidade-Empresa no Brasil: evidências do Diretório dos Grupos de Pesquisa no Brasil." Estudos Econômicos, 37(2); p. 212-233.

ROSENBERG, N. (1992). "Scientific instrumentation and university research?" Research Policy, 21(4); p. 381-390.

RUIZ, A. U.; BHAWAN, R. (2010). "Diferenças de comportamento inovador entre empresas nacionais e estrangeiras no Brasil." Revista Brasileira de Inovação, 9(1); 29-68.

STRACHMAN, E.; AVELLAR, A. P. (2008). "Estratégias, Desenvolvimento Tecnológico e Inovação no Setor de Bens de Capital no Brasil.” Ensaios FEE, 29; p. 237-266.

SUZIGAN, W. ; ALBUQUERQUE, E. (2008). “A interação entre universidades e empresas em perspectiva histórica no Brasil.” Texto para discussão 329. Belo Horizonte: Cedeplar/UFMG.

UNITED NATIONS CONFERENCE ON TRADE AND DEVELOPMENT (2005). World investment report: transnational corporations and internationalization of R\&D. Genebra: Nações Unidas. 
SILVA NETO, F. C. C. et al. A interação universidades/institutos públicos de pesquisa... 